\title{
Advent of synthetic life
}

\author{
Researchers synthesized the first \\ functional synthetic bacterial \\ genome; repeating this feat with dif- \\ ferent bacteria will require further \\ methodological development. \\ Recently, scientists from the J. Craig \\ Venter Institute (JCVI) published the \\ first report of a functional synthetic \\ genome (Gibson et al., 2010). The \\ work elicited a wide range of respons- \\ es in the scientific and popular press, \\ from hailing the achievement as 'the \\ crossing of the Rubicon', as stated in \\ the The Economist, where the editors
} predicted that "it is now possible to conceive of a world in which new bacteria (and eventually, new animals and plants) are designed on a computer and then grown to order," to voices cautioning that reproducing life is not the same as understanding and thus controlling it.

It is far too early to say where exactly this work will lead, but it is indeed a methodological feat that required overcoming many hurdles. Some hurdles were cleared in work described previously by researchers at the JCVI. In 2008, they reported the synthesis and cloning of the almost 600-kilobase (kb) Mycoplasma genitalium genome but could not show that the genome was actually functional because they had no way of transplanting it into another bacterium. The scientists transplantated a cloned genome in 2009 when they shuttled the genome of M. mycoides into yeast, modified it and put it back into $M$. capricolum, converting $M$. capricolum to M. mycoides.

The ultimate goal was, of course, not to shuttle natural genomes back and forth but to transplant a synthetic genome. The researchers did not pursue this plan with the synthetic $M$. genitalium because they lacked a genome transplantation protocol, and a colony takes more than four weeks to form. Instead, they settled on $M$. mycoides as their blueprint, ordered 1,100 chemically synthesized building blocks of

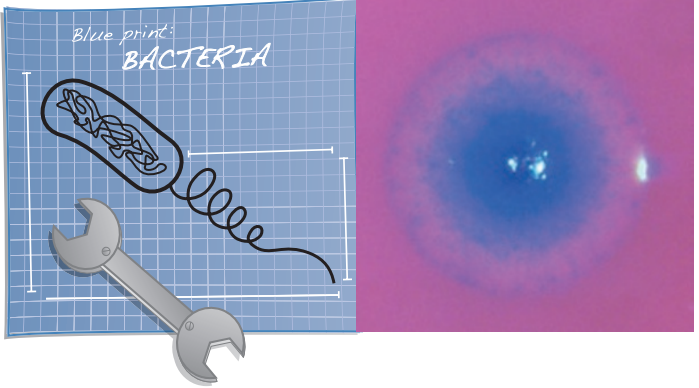

Synthetic M. mycoides clone. Image courtesy of D. Gibson. Cartoon by Katie Vicari.

$1 \mathrm{~kb}$ each and intended to assemble them in yeast in a two-step process.

The problem with this strategy was that, as Daniel Gibson, first author on the paper describing the synthetic genome, puts it, they had too much confidence in yeast. The scientists knew from their cloning of the M. genitalium genome that yeast could take up large fragments and assemble them correctly, but for M. mycoides the two-step assembly process failed. They decided to take it more slowly and first assemble 10 -kb fragments in yeast, then transform the DNA into Escherichia coli to amplify it. And in doing so, they promptly ran into the next roadblock.

Gibson recalls, "it was amazing; with the M. genitalium synthesis we were propagating half genomes, which were almost $300 \mathrm{~kb}$ in E. coli, and we were getting lots of DNA, but then just $10 \mathrm{~kb}$ with this genome were really difficult." The scientists persisted and eventually assembled all eleven 100-kb fragments in yeast but could amplify none of them in E. coli. The only way forward was to use yeast as a production as well as an assembly vessel. The researchers followed a protocol they expected to succeed. It did not. They were concerned that their project hit a dead end, but the solution turned out to lie in highly purified DNA because even the slightest contamination from yeast chromosomes interfered with the assembly.
To ensure that their 100-kb building blocks were functional, the JCVI team first tested each of them in a semisynthetic assembly; they combined each of the eleven blocks with the rest of the natural genome and monitored colony growth. This mixand-match strategy allowed them to correct a point mutation in an essential gene in one of the fragments; after that, the coast was clear for the assembly of the synthetic genome and its transplantation.

Now, Gibson says, the research will split in two directions. One team will work on creating a minimal cell, stripping each of the 100-kb fragments down to the bare essentials and then combining them again to form a minimal $M$. mycoides. The other endeavor will be to lay the groundwork for other synthetic genomes. Theoretically, the approach used for M. mycoides should work for other bacteria as well; in practice, it may not be so straightforward.

One question is how big and $(\mathrm{G}+\mathrm{C})$ rich of a genome can be synthesized and assembled in yeast. As the largest yeast chromosome is 2 megabases, Gibson speculates that a genome of this size can be amplified. Another question is how easily another genome can be transplanted. "Right now," says Gibson, "we have only one pair of organisms that anybody has ever demonstrated genome transplantation with. If we want to transplant say, cyanobacterial genomes, we have to develop new transplantation systems."

Judging by the reaction to the first synthetic cell, the spotlight will certainly be on these developments.

\section{Nicole Rusk}

\section{RESEARCH PAPERS}

Anonymous. And man made life. The Economist (22 May 2010).

Gibson, D.G. et al. Creation of a bacterial cell controlled by a chemically synthesized genome. Science advance online publication (20 May 2010). 